The five variables considered were subjected to an analysis of variance taking into account the herd effects ( 18 levels), the litter order (3 levels), the genotype $(2$ levels) and interaction effects between these factors. The interaction herd $\times$ genotype was significant $(\mathrm{P}<0.01)$ for numbers of piglets born and weaned per litter. The effect of genotype was significant $(P<0.01)$ for the other three variables. In " $1 / 2$ Chinese » sows, the number of piglets born, born alive and weaned per littter was improved by $2.9,2.9$ and 2.0 units, respectively and the weaning-fertilization and farrowing intervals were reduced by 5.4 and 4.1 days as compared to European contemporaries. This leads to an average of weaned piglets/sow/year of reproductive life of 5.4 units, with nevertheless large variations between herds $(-2$ to $+10 \mathrm{piglets} / \mathrm{sow} / \mathrm{year})$.

Fattening and carcass performance of $76 \ll 1 / 4$ Chinese $»$ and 77 control pigs sired by 36 terminal boars in the previous herds were then compared in central progeny testing stations. The analysis of variance took into consideration the control batch ( 6 levels), the genotype ( 2 levels), the sire ( 36 levels) as well as the slaughter weight for the cutting criteria and the slaughter date for the meat quality criteria. Daily mean gain of $\ll 1 / 4$ Chinese » pigs was reduced by $22 \mathrm{~g}$ and food conversion ratio was increased by $0.07 \mathrm{~kg}$ feed $/ \mathrm{kg}$ gain. These differences were not significant. On the other hand, carcass weight was reduced by $0.7 \mathrm{~kg}$, muscle percentage by $4.2 \mathrm{p} .100$ and fat percentage increased by $4.6 \mathrm{p} .100$ in $~ 1 / 4$ Chinese » pigs. Those differences were significant $(P<0.01)$. On the whole, meat quality criteria were in favour of « $1 / 4$ Chinese» and showed an improvement in the meat quality index (MQI) of 0.8 point $(\mathrm{P}<0.05)$.

A total of 73 hams $(34$ controls and $39 \ll 1 / 4$ Chinese $»)$ were processed into «Paris ham». The handicap of 3.7 p. $100(\mathrm{P}<0.01)$ concerning the anatomical yield of $\ll 1 / 4$ Chinese $\gg$ hams was only partly compensated by a higher technological yield $(+1.1$ p. 100$)$ so that the final yield was reduced by 2.5 p. $100(\mathrm{P}<0.01)$.

An approximate economic balance showed that a minimum productivity gain of 6 piglets weaned per year in $~ 1 / 2$ Chinese » sows is required to compensate for the reduction in the gross margin recorded in a $\ll 1 / 4$ Chinese » terminal product.

\title{
Polymorphism of the major histocompatibility complex SLA in several pig breeds in France
}

\author{
Christine RENARD *, M. LUQUET **, P. GOULLIEUX ***, M. VAIMAN ***** \\ *I.N.R.A., Laboratoire de Radiobiologie Appliquée, 78350 Jouy-en-Josas \\ **I.T.P., 149, rue de Bercy, 75595 Paris Cedex 12 \\ *** I.T.P. Région Nord, Station de Sélection Porcine \\ 27, rue de Bapaume, Le Transloy, 62450 Bapaume \\ **** Commissariat à l'Energie Atomique-DPS-SPE-LRA, 91191 Gif-sur-Yvette
}

The major histocompatibility complex SLA was tested in several breeds including highly selected breeds and local breeds. The influence of the founding effect, mutation and putative selection upon the frequency of SLA alleles in all these breeds was observed. The genetic drift was also investigated in small populations corresponding to local breeds. Because of its high polymorphism, SLA complex seemed well fitted for these observations.

Preliminary results showed significant correlations between some SLA alleles and production traits such as growth rate and backfat thickness. The frequency of SLA alleles associated to unfavourable production traits decreased during the 6-year testing period. By contrast, favourable SLA haplotypes increased significantly.

Massive introduction of English and Dutch breeding animals also contributed to modify SLA distribution.

Comparison of the SLA haplotypes between the different breeds showed, unexpectedly, that Pietrain are closer to Large White than to Landrace. In local breeds, there is a rather large polymorphism in spite of the small number of animals available. As compared to selected breeds, no gametic association was observed in the local breeds. 